\title{
KHK-Patienten sollten sich von Raucherecken fernhalten
}

- Wenn am Arbeitsplatz und in Restaurants und Bars nicht mehr geraucht werden darf, gibt es deutlich weniger Herzinfarkte, bestätigt eine aktuelle US-Studie. Danach ist Passivrauchen ein wichtiger vermeidbarer Risikofaktor für einen Herzinfarkt.

In Olmstedt County in Minnesota wurde im Jahr 2002 in Restaurants und im Jahr 2007 auch in Bars und an allen Arbeitsplätzen das Rauchen verboten. Eineinhalb Jahre später lag die Herzinfarktrate in der Bevölkerung um 33\% niedriger als vor Einführung der Gesetze zum Nichtraucherschutz, wie Ärzte um Dr. Richard D. Hurt von der Mayo Clinic in Rochester jetzt berichten.

Die Mediziner hatten die Krankenakten aus den beiden Krankenhäusern des Landkreises nachträglich ausgewertet. In den 18 Monaten vor dem ersten Antirauchergesetz hatten sich pro 100000 Einwohner 150,8 Herzinfarkte ereignet, in den 18 Monaten nach dem zweiten Gesetz waren es nur noch 100,7 ( $<<0,001$ ). Das Rauchverbot in Restaurants allein hatte dagegen nicht zu

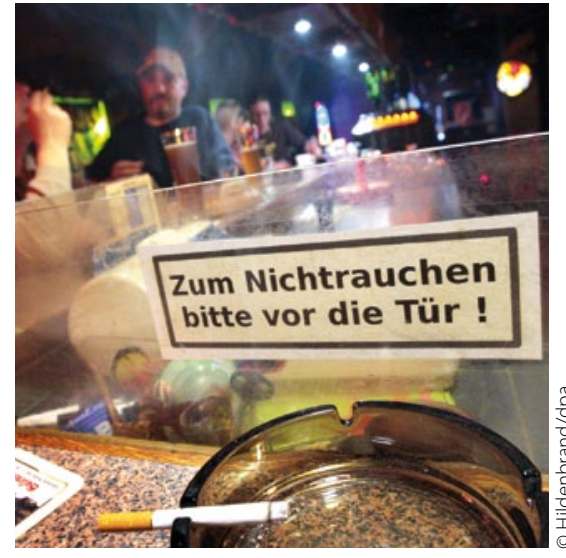

Auch KHK-Patienten sollten hier lieber draußen bleiben.

einem Rückgang von Herzinfarkten geführt. Die Befürchtung, dass bei einem Rauchverbot in öffentlichen Räumen verstärkt zu Hause gequalmt wird, bestätigte sich nicht. Im Gegenteil: Der Pro-Kopf-Verbrauch an Zigaretten wie auch die Zahl der Raucher ging zurück. Andere Ursachen für die dras- tische Absenkung der Herzinfarktrate schließen Witt et al. weitgehend aus: Die Prävalenzraten von Bluthochdruck und $\mathrm{Hy}$ percholesterinämie blieben im Studienzeitraum unverändert, die von Adipositas und Diabetes nahmen sogar zu.

Die US-Studie bestätigt andere Studien, in denen nach Einführung von Rauchverboten ebenfalls verminderte Herzinfarktraten festgestellt wurden. Nach einer Metaanalyse von 17 Studien gehen infarktbedingte Klinikeinweisungen um $10 \%$ zurück (Mackay DF et al., Heart 2010).

Laut Witt et al.,„sollte Passivrauchen zusätzlich zu einer positiven Familienanamnese, Bluthochdruck, Hyperlipidämie, Diabetes und Bewegungsmangel als Hauptrisikofaktor für einen Herzinfarkt angesehen werden“. Und weiter: „Jeder sollte soweit wie möglich das Passivrauchen vermeiden, KHK-Patienten sollten sich dem gar nicht aussetzen."

BS =

Hurt RD et al. Arch Intern Med 2012; online 29. October; doi:10.1001/2013.jamainternmed.46

\section{Nutzenbewertung: Ein Unwort!}

Kaum ein Wort wird in der Medizin derzeit so strapaziert wie der Begriff "Nutzenbewertung". Es eignet sich durchaus als Unwort des Jahres. Neue Medikamente sollen sogar einen Mehrnutzen bringen, und dies sei, so die Autoren des alljährlich erscheinenden Verordnungsreports, bei über der Hälfte aller neu zugelassenen Medikamente nicht der Fall. Diese Aussage ist schon deshalb unrichtig, weil die Hersteller ja in jedem Fall einen Zusatznutzen, sprich Gewinn erwarten dürfen.

Doch da fragt man sich schon, was dieser Unsinn eigentlich soll. Haben Sie sich etwa bei dem Kauf von ein Paar neuen Schuhen oder gar einer neuen Unterhose auch schon einmal gefragt, ob das Neue für Sie einen wirklichen Zusatznutzen bringt? Würden Sie auf eine solche Neuanschaffung etwa verzichten, wenn die neue Unterhose nicht besser funktioniert als die alte?

So sollten wir es bei den Medikamenten wohl auch sehen. Es ist doch einleuchtend, dass Patienten nicht immer dasselbe, sondern mal etwas Anderes schlucken möchten, nicht immer die blaue, sondern vielleicht jetzt eine rosa Tablette oder eine runde statt einer eckigen unabhängig davon, ob die nun mehr bringt als die alte. Solchen optischen und sicherlich auch pharmakologischen Abnutzungserscheinungen muss man entgegenwirken, indem man immer wieder etwas Neues auf den Markt bringt, und dies darf dann auch etwas teurer sein. Zusatznutzen hin oder her! Diese Botschaft hat die Pharmaindustrie erfreulicherweise richtig verstan- den, sodass sie uns ständig mit "Neuem" auch Innovationen genannt - beglückt. Dafür sollte man ihr dankbar sein und sie nicht ständig mit unsinnigen Forderungen nach Mehrnutzen verärgern; denn nur so lässt sich die viel beschworene PatientenCompliance nachhaltig sichern. Was neu ist, ist auch sexy, ob es mehr kann oder nicht, muss niemanden interessieren und sollte auch nicht unbedingt überprüft oder gar kritisch hinterfragt werden.

Dies gilt übrigens auch für zwischenmenschliche Beziehungen. Wer sein Privatleben nach dem Konzept der seriellen Monogamie gestaltet, sollte keinesfalls die Frage nach dem Mehrnutzen stellen, denn beim nächsten neuen Mann oder der nächsten neuen Frau wird es auch nicht besser.

Dr. med. Peter Stiefelhagen - 